\title{
FracL: A Tool for Characterizing the Fractality of Landscape Gradients from a New Perspective
}

\author{
Peichao Gao ${ }^{1,2,3,4} \mathbb{D}$, Samuel A. Cushman ${ }^{5}$, Gang Liu ${ }^{6}$, Sijing Ye ${ }^{1}{ }^{\mathbb{D}}$, Shi Shen ${ }^{1}(\mathbb{D}$ \\ and Changxiu Cheng $1,2,3,4, *$ \\ 1 State Key Laboratory of Earth Surface Processes and Resource Ecology, Beijing Normal University, Beijing \\ 100875, China; gaopc@bnu.edu.cn (P.G.); yesj@bnu.edu.cn (S.Y.); shens@mail.bnu.edu.cn (S.S.) \\ 2 Key Laboratory of Environmental Change and Natural Disaster, Beijing Normal University, \\ Beijing 100875, China \\ 3 Faculty of Geographical Science, Beijing Normal University, Beijing 100875, China \\ 4 Center for Geodata and Analysis, Beijing Normal University, Beijing 100875, China \\ 5 US Forest Service, Rocky Mountain Research Station, Flagstaff, AZ 86011, USA; scushman@fs.fed.us \\ 6 College of Earth Sciences, Chengdu University of Technology, Chengdu 610000, China; liuganggis@sina.com \\ * Correspondence: chengcx@bnu.edu.cn; Tel.: +86-10-5880-7241
}

Received: 13 August 2019; Accepted: 20 October 2019; Published: 22 October 2019

\begin{abstract}
The past several years have witnessed much progress in landscape ecology and fractal analysis. In landscape ecology, the gradient model of landscape patterns (i.e., landscape gradient) has emerged as a new operating paradigm, where most landscape metrics do not apply because they were developed for the patch mosaic model. In the fractal analysis, a new definition of fractal has been proposed, and various new fractal metrics have been developed. This technical note aims to provide an intersection of these two lines of advance, which will further present an opportunity to advance geo-informatics by considering the latest progress in both landscape ecology and fractal analysis. We first present an overview of the new definition of fractal and all the fractal metrics developed under this new definition. Since the chief obstacle to geographers and landscape ecologists in applying these metrics is the lack of readily accessible methods for their easy computation, we then develop an integrated tool to compute them on landscape gradients. The developed tool facilitates the computation of these new fractal metrics. A case study was carried out with real-life landscape gradients, namely a digital terrain model. These new fractal metrics and the developed tool can be expected to facilitate the fractal characterization of the patterns of gradient landscapes and the understanding of landscape dynamics from a new perspective.
\end{abstract}

Keywords: fractal analysis; fractality; landscape pattern; landscape gradient

\section{Introduction}

Recent years have seen substantial innovation in both landscape ecology [1,2] and fractal analysis. For example, in landscape ecology, the operating paradigm has been extended from patch mosaics [3] to landscape gradients $[4,5]$. In the patch-mosaic paradigm, a landscape is modelled as a mosaic of discrete patches by either subjectively or objectively categorizing the environmental attribute of interest (e.g., forest community types, ground temperature, and biodiversity). By contrast, in the gradient paradigm, a landscape is modelled as a continuous surface or a series of surfaces of an environmental attribute, usually represented as a matrix of quantitative value. This gradient paradigm turns out to be more appropriate in analyzing continuously varying landscape patterns because there is no need to transform a continuous (or quantitative) attribute of interest into categorical (or qualitative) and thus no loss of information caused by the transformation. For example, Evans and Cushman [6] 
found it more appropriate to model vegetation patterns using the gradient paradigm in studying the occurrence probability of individual species. The challenge to operationalizing this gradient paradigm, however, is to develop metrics and tools for analyzing the structure of landscape gradients from diverse aspects, given that many existing landscape metrics are designed only for patch mosaics, e.g., [7]. Landscape ecologists are making great efforts to address this challenge [8,9]. Recent milestones include the introduction of surface metrics from surface metrology to landscape ecology [10-12] and the development of an R package for computing surface metrics [13].

Concurrently, in fractal analysis, the definition of fractal has continuously evolved since its original description at the beginning of the last century. At that time, the fractal was understood as some mathematical constructions such as the Koch curve [14], Sierpinski carpet [15], and Menger sponge [16]. These constructions display strict self-similarity at multiple characteristic scales. However, such constructions are purely theoretical models and do not exist in nature. Later, the definition of fractal was relaxed by Mandelbrot [17] to include statistical self-similar constructions, a well-known example under this definition is the British coastline [18]. In this definition, the statistical self-similarity must be observed based on the power-law relationship established between the measurement scale and the measured length/area/volume. However, such a relationship is still "too strict for many geographic features" [19], making the computation of fractal dimension challenging. This challenging issue was also noted in landscape ecology. For example, Vranken, et al. [20] described the computation of fractal dimension as a "practical difficulty" (p. 62), and Parrott, et al. [21] noted that "while well-defined for perfect mathematical objects, when applied to real objects, the fractal dimension is a problematic measure, since it is only applicable over a limited range of scales" (p. 351). To address this challenging issue, a new definition of fractal has been proposed [19] by relaxing the mathematical constraint of self-similarity from a power-law distribution [18] to a heavy-tailed formulation [22].

This new definition has been increasingly accepted and successfully applied to many fields such as cartographic generalization [23], big data analysis [24], and spatial data visualization [25]. However, it has yet to be applied or explored in the context of landscape ecology, although in this field fractal analysis has long been used, e.g., [7,26-28]. On the other hand, the fractal is useful for landscape ecology. It is scale-independent and thus can be used to perform cross-scale analysis of landscape patterns and processes [29]. Therefore, introducing the new definition of fractal to landscape ecology will provide geographers and ecologists with an alternative way to characterize the fractality (i.e., fractal property) of geo-data in general and landscape gradients in particular. However, the practical difficulty therein is the lack of a tool to facilitate the computation of diverse fractal metrics that have been proposed under this new definition. This study is aimed at developing such a tool, by which to facilitate the use of and to promote the improvement of these fractal metrics.

\section{The New Definition of Fractal}

The new definition of a fractal can be summarized as follows: "a feature is thought to be fractal if the scaling pattern of 'far more small things than large ones' can be observed multiple times" [30]. There are three keys to understanding this new definition: the notion of and the scaling pattern of "far more small things than large ones", and how this pattern can be observed multiple times.

The notion of "far more small things than large ones" was first clearly formulated by Jiang [31] and has become widely used in recent years, e.g., [32-36]. The terms "small things" and "large ones" of this notion can be understood broadly. For example, there are far more small earthquakes than large ones on Earth [37], and there are far more low-mass stars than massive ones in the Universe [38]. In practice, "small things" and "large ones" are distinguished according to a quantitative attribute of these things by using a classification method called head/tail breaks [22]. The head/tail breaks method divides the values of the attribute into a head part and a tail part, according to the average of these values. The head part consists of those values that are greater than the average, and the corresponding things are referred to as "large ones". By contrast, the tail part contains the rest of the values and corresponds to "small things". For instance, if the average magnitude of earthquakes worldwide is 5.0, 
then the earthquakes with a magnitude of 5.0 or less are the "small things" and the rest are the "large ones".

When it comes to the scaling pattern of "far more small things than large ones", a range of scales should be used in measuring a feature (a scaling pattern is sometimes referred to as a scaling relation) [39,40]. Let us take the classical Koch [14] curve, as shown in Figure 1, as an example and assume that the length of its shortest segment is $1 / 9$ unit. In this example, one can observe as many as 16 line segments (referred to as Segments A) when measuring the Koch curve using a scale of 1/9 unit, or a total of 4 line segments (Segments B) using a scale of $1 / 3$ unit, or only 1 line segment (Segment C) using a scale of 1 unit. All these 21 line segments exhibit a typical scaling pattern of "far more small things than large ones", which can be stated as follows: there are far more short line segments than long ones.

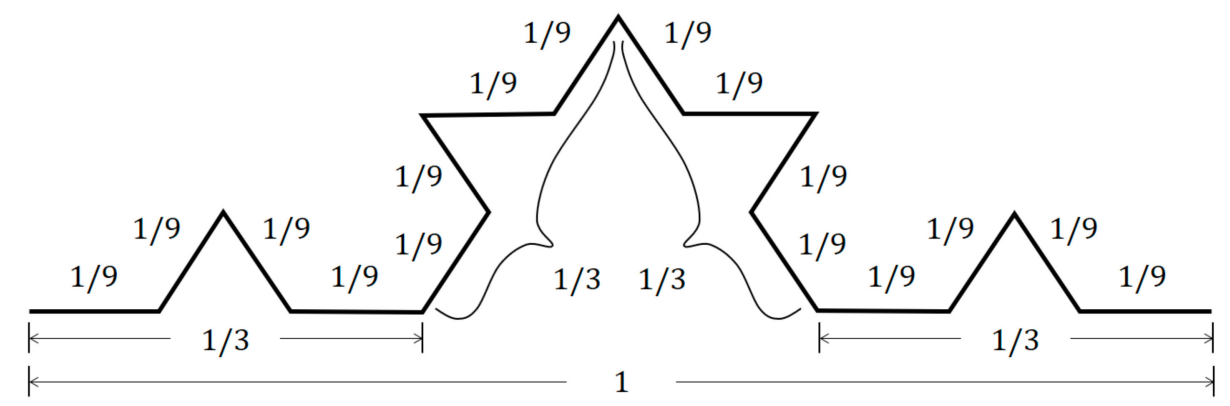

Figure 1. Koch curve, where the length of the shortest line segment is $1 / 9$ unit.

The scaling pattern of "far more small things than large ones" can sometimes be observed multiple times, by iteratively removing "small things". For instance, this pattern can be observed twice with the 21 line segments (Figure 1). The first observation has been described in the preceding paragraph, where the "small things" (i.e., short line segments) refer to the 16 Segments A and the "large ones" (i.e., long line segments) contains both the 4 Segments B and the Segment C. When the 16 Segments A are removed from the 21 line segments, the scaling pattern of "far more small things than large ones" can still be observed: The new "small things" refer to 4 Segments B, while the new "large one" is the Segment $C$.

It should be noted that this new definition of a fractal is focused on the attributes of a feature, rather than the spatial pattern or geometric shape. For example, the focus has been placed on the lengths of line segments in Figure 1, rather than the spatial pattern formed by these line segments. In this sense, it seems more suitable to refer to such a definition as fractal attribute. By contrast, the conventional definition of fractal is focused on the spatial pattern or geometric shape of a feature. This is why it is also widely referred to as fractal geometry [41,42].

\section{Overview of Metrics under the Latest Definition of Fractal}

Six metrics have been proposed for characterizing a fractal under this new definition. These fractal metrics are ht-index, the cumulative rate of growth (CRG index), the ratio of areas in a rank-size plot (RA index), two unified metrics (UM1 and UM2), and the fractional ht-index (fht-index).

\subsection{Ht-Index}

Ht-index [19] was the first metric proposed under the new definition of fractal, and it is straightforward. The ht-index of a fractal equals one plus the number of observations of the scaling pattern of "far more small things than large ones", or, in other words, it equals one plus the maximum number of times that the head/tail breaks method can be iteratively applied. As such, ht-index is also called "head/tail breaks index" by Chen [34] and Huang [43]. 
In practical computation, the value of ht-index is affected by how one confirms an observation of "far more small things than large ones" or, more precisely, by the threshold percentage of "small things" therein. Scholars have used different threshold percentages of small things, such as 33\% [44], 40\% [45], and $50 \%[46,47]$, but it is important to note that a comparison between two ht-indexes is meaningful only if they are computed based on the same threshold.

The essence of the ht-index of a fractal is the number of levels of the fractal's underlying hierarchy, which is formed by iteratively applying the head/tail breaks method. Note that the term "hierarchy" here does not have the same meaning as that in the hierarchy theory $[48,49]$. For example, the ht-index of the Koch curve in Figure 1 is three, revealing that there are three levels: a bottom level of 16 Segments A, a medium level of 4 Segments B, and a top level of 1 Segment C. These three levels form a hierarchy.

The advantage of ht-index is that it is easy to interpret. This is because it indicates the number of observations of the scaling pattern of "far more small things than large ones". Note that this number of observations is the key to evaluate the fractality of a feature [19]. However, the problem of ht-index is that it is not sensitive in capturing the evolution of a fractal. In other words, ht-index is not capable of distinguishing two similar fractals if only the two fractals have the same number of underlying hierarchical levels. For example, both curves in Figure 2 have a ht-index of three.

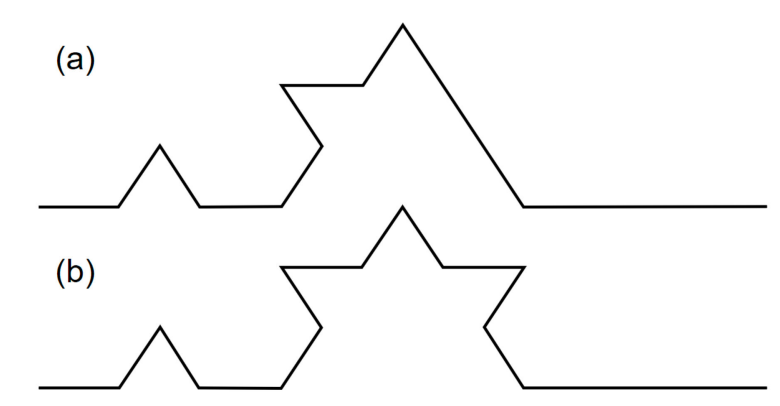

Figure 2. Two curves with the same ht-index. The first curve (a) is less similar to the Koch curve than is the second curve (b)

\subsection{CRG Index}

To address the insensitivity issue of ht-index, Gao, et al. [50] developed an improved index called the cumulative rate of growth (CRG index). Its formula is as follows:

$$
\mathrm{CRG}=\sum m_{i+1} / m_{i}
$$

where $m_{i}$ denotes the $i$-th average calculated in performing the head/tail breaks method.

To illustrate the core idea of the CRG index, let us consider again the two curves shown in Figure 2. For the first curve, one can observe 14 Segments A, or 4 Segments B, or 1 Segment C. The head/tail breaks method can be performed twice on these segments, using an average of $35 / 9$ (i.e., the average length of all segments) for the first time and an average of 7/3 (i.e., the average length of Segments B and $C$ ) for the second time. By contrast, when dealing with the second curve, the numbers of Segments A, B, and C are 15, 4, and 1, respectively. The two averages for performing the head/tail breaks method are 4 and $7 / 3$. Although the averages used when dealing with the two curves are different, these two curves have the same ht-index, namely 3 . However, the CRG index is capable of distinguishing the fractalities of the two curves: The CRG index of the first curve is $(35 / 9) /(7 / 3)=5 / 3$, whereas that of the second curve is $(4) /(7 / 3)=12 / 7$.

Compared with ht-index, the advantage of the CRG index is that it captures fractal evolution in a more sensitive way, as demonstrated by the two curves in Figure 2. In other words, the CRG index is capable of distinguishing two similar fractals. This advantage means that the CRG index should be more applicable to landscape ecology in reflecting the dynamics of landscape patterns. However, 
the disadvantage of CRG index is that its value no longer indicates the number of levels of the fractal's underlying hierarchy, which is a meaningful interpretation of fractal.

\subsection{RA Index}

RA index [51] is an alternative to the CRG index in addressing the insensitivity issue of ht-index. Its core idea is to measure how heavy-tailed the scaling pattern of "far more small things than large ones" is. Essentially two steps are involved in computing the RA index of a feature.

The first step is to display all the "things" (i.e., small things and large ones) in a plot where the $y$-axis represents a quantitative attribute of these things and the $x$-axis is the rank of a thing according to its value of the attribute. For example, the resultant plot of the Koch curve is shown in Figure 3. Such a plot of scatter points is commonly referred to as a rank-size plot.

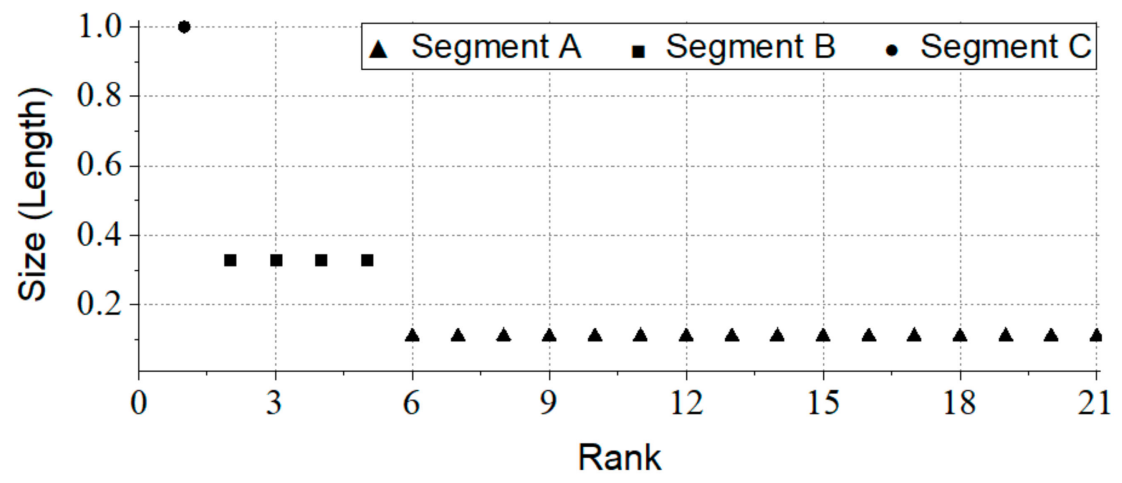

Figure 3. The rank-size plot of the line segments observed with the Koch curve.

The second step is to compute the areas of two polygons constructed in the rank-size plot. One polygon, as shown by $S_{1}$ in Figure 4 , is constructed by firstly drawing a parallel line to the $y$-axis (i.e., size-axis) through the first scatter point, then a parallel line to the $x$-axis (i.e., rank-axis) through the last scatter point, and finally a polyline to connect each scatter point. The other is constructed by drawing a straight line to connect the first and the last scatter point, as shown by $S_{2}$ in Figure 4. The value of the RA index is computed as a ratio of areas:

$$
\operatorname{RA}=\operatorname{Area}\left(S_{1}\right) /\left(\operatorname{Area}\left(S_{1}\right)+\operatorname{Area}\left(S_{2}\right)\right)
$$

where $\operatorname{Area}()$ is a function that returns the area of a polygon.

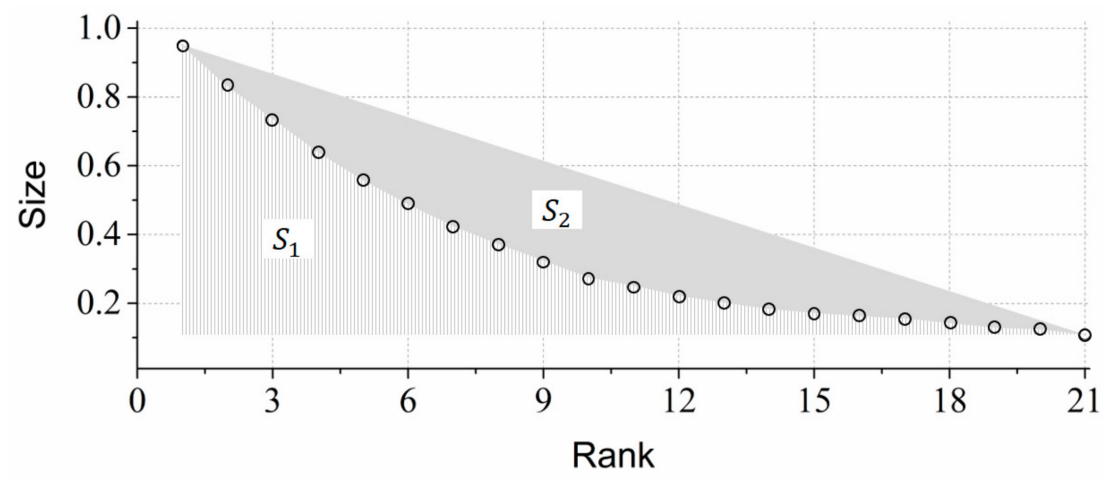

Figure 4. Two polygons constructed for computing the RA index of a feature.

Take the two curves in Figure 2 as examples again. For the first curve, its $\operatorname{Area}\left(S_{1}\right)$ and $\operatorname{Area}\left(S_{2}\right)$ are 1.3332 and 6.6669 , respectively, so its RA index is computed as 1.3332/6.6669 $=0.1666$. For the second 
curve, its $\operatorname{Area}\left(S_{1}\right)$ and $\operatorname{Area}\left(S_{2}\right)$ are 1.3332 and 7.1113, respectively, so its RA index is computed as $1.3332 / 7.1113=0.1579$.

RA index has the same advantages and disadvantages as the CRG index. However, its unique characteristic is that it is not computed by performing the head/tail breaks method. Instead, its computation relies on the pattern displayed in a rank-size plot.

\subsection{Unified Metrics}

Unified metrics [30] were proposed to combine the advantages of both the ht-index and CRG/RA index. Such a combination was achieved by answering the following two questions:

- "I know there are far more small things than large ones, but how small (or large)?"

- "I know there are far more small things than large ones, but how many more?"

The answers to these two questions are shown in Formulas (3) and (4), respectively.

$$
\begin{gathered}
y_{1}=\left\{\begin{array}{c}
1-m_{\text {all }} / m_{\text {head }}, \exists m_{\text {head }} \\
0, \nexists m_{\text {head }}
\end{array}\right. \\
y_{2}=\left\{\begin{array}{c}
n_{\text {tail }} / n_{\text {all }}, n_{\text {tail }} \neq n_{\text {all }} \\
0,
\end{array}\right.
\end{gathered}
$$

where $m_{\text {all }}$ and $m_{\text {head }}$ are the average size of all "things" and that of "things" which are classified as the head, respectively. The parameters $n_{\text {all }}$ and $n_{\text {tail }}$ denote the total number of all things and that of the things which are classified as the tail, respectively.

Then, ht-index was redefined as a decimal number, of which the whole-number part equals the original ht-index to indicate the number of observations of the scaling pattern of "far more small things than large ones". The decimal part equals the result of either Formula (3) or (4), resulting in two different unified metrics, referred to as UM1 and UM2, respectively. For example, the (UM1)s for the first and the second curve in Figure 2 are 3.5614 and 3.5714, respectively. The (UM2)s for the first and the second curve in Figure 2 are 3.7368 and 3.7500, respectively.

\subsection{Fht-Index}

Fht-index [52] is an alternative to unified metrics. In developing fht-index, two new concepts were introduced, namely whole and sub-whole. A whole is defined as a group of numbers whose ht-index will decrease by one if the smallest number (or one of the smallest numbers, if there is more than one) is removed from the group. A sub-whole is defined as a group of $n\left(n \in \mathrm{Z}^{+}\right)$numbers that satisfy the following conditions: the group itself is a whole, and it consists of the largest $n$ numbers of another whole. A typical whole and its sub-wholes are shown in Table 1 . In this case, the ht-index of the whole equals four, and it will decrease to three if the smallest number (i.e., 1/39) is removed. Similarly, if the smallest number is removed from either of the sub-wholes, the ht-index of that sub-whole will also decrease by one.

Table 1. A typical whole, the sub-wholes belonging to the whole, and their ht-indices.

\begin{tabular}{ccc}
\hline & Numbers & Ht-Index \\
\hline Whole & $\left\{1, \frac{1}{2}, \frac{1}{3}, \frac{1}{4}, \frac{1}{5}, \frac{1}{6}, \frac{1}{7}, \frac{1}{8}, \frac{1}{9}, \frac{1}{10}, \frac{1}{11}, \frac{1}{12}, \frac{1}{13}, \cdots, \frac{1}{38}, \frac{1}{39}\right\}$ & 4 \\
Sub-whole 1 & $\left\{1, \frac{1}{2}, \frac{1}{3}, \frac{1}{4}, \frac{1}{5}, \frac{1}{6}, \frac{1}{7}, \frac{1}{8}, \frac{1}{9}\right\}$ & 3 \\
Sub-whole 2 & $\left\{1, \frac{1}{2}, \frac{1}{3}\right\}$ & 2 \\
\hline
\end{tabular}

Note that $50 \%$ was used as the threshold in computing ht-index.

Fht-index is computed according to the following rules. First, the fht-index of a whole equals the ht-index of the whole. Second, for a data set that is not a whole, its fht-index is interpolated 
between the ht-index of the whole to which the data set belongs and that of the largest sub-whole (i.e., the sub-whole which has the most members) belonging to the data set. To concretely illustrate this idea, let us consider the four data sets shown in Table 2. All these data sets have the same whole and the same largest sub-whole. In this case, their fht-indices are derived by linear interpolation between the ht-indices of the whole and the largest sub-whole. It is worth noting that Jiang and Ma [52] utilized a power interpolation instead. Take the two curves in Figure 2 for another example. The first curve has an $\mathrm{ft}$-index of 3.0625, while the second has an $\mathrm{ft}$-index of 3.0681 .

Table 2. Four data sets and their whole, largest sub-whole, ht-index, fht-index.

\begin{tabular}{cccc}
\hline & Numbers & Ht-Index & Fht-Index \\
\hline Whole & $\left\{1, \frac{1}{2}, \frac{1}{3}, \frac{1}{4}, \frac{1}{5}, \frac{1}{6}, \frac{1}{7}, \frac{1}{8}, \frac{1}{9}\right\}$ & 3 & 3.0 \\
Data set 1 & $\left\{1, \frac{1}{2}, \frac{1}{3}, \frac{1}{4}, \frac{1}{5}, \frac{1}{6}, \frac{1}{7}, \frac{1}{8}\right\}$ & 3 & 2.8 \\
Data set 2 & $\left\{1, \frac{1}{2}, \frac{1}{3}, \frac{1}{4}, \frac{1}{5}, \frac{1}{6}, \frac{1}{7}\right\}$ & 3 & 2.6 \\
Data set 3 & $\left\{1, \frac{1}{2}, \frac{1}{3}, \frac{1}{4}, \frac{1}{5}, \frac{1}{6}\right\}$ & 3 & 2.4 \\
Data set 4 & $\left\{1, \frac{1}{2}, \frac{1}{3}, \frac{1}{4}\right\}$ & 3 & 2.2 \\
Largest sub-whole & $\left\{1, \frac{1}{2}, \frac{1}{3}\right\}$ & 2 & 2.0 \\
\hline
\end{tabular}

Note that $50 \%$ was used as the threshold in computing ht-index.

\section{Development of a Software Tool: FracL}

A practical challenge to implementing fractal analysis from this new perspective lies in the lack of tools to readily compute the fractal metrics summarized in the previous section. To address this need, we developed a software tool (referred to as FracL) in this study to facilitate the computation of these fractal metrics on landscape gradients (Figure 5). The functional design, algorithmic considerations, and technical implementation of the software tool are described in this section.

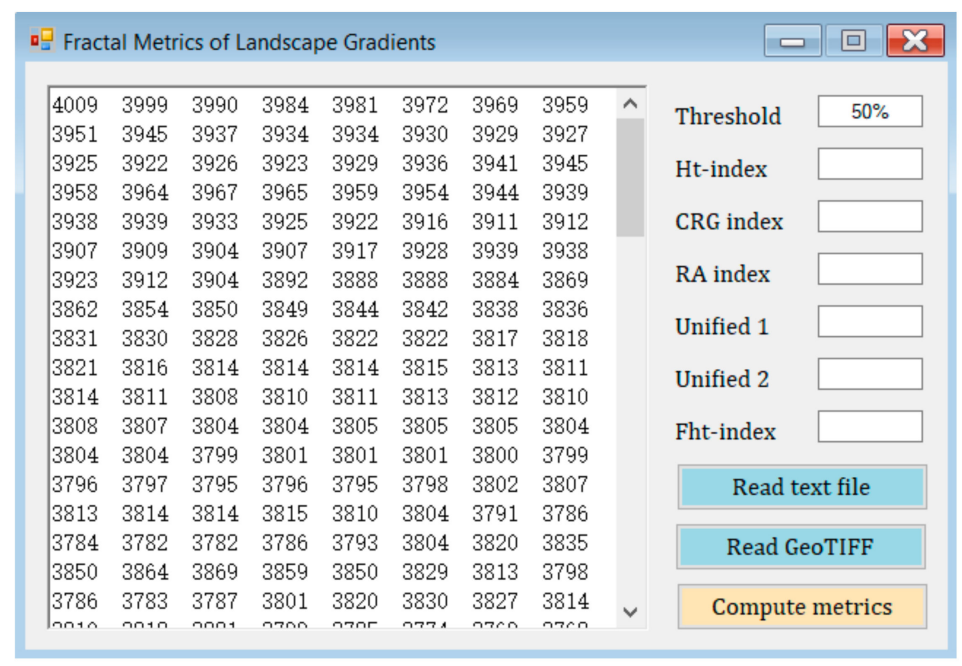

Figure 5. The developed tool for easily computing fractal metrics.

The software tool is designed to perform the following functions. The first is an input-output function. The tool should be capable of (a) reading a landscape gradient from the standard input (i.e., the keyboard), (b) reading a series of landscape gradients from data files, and (c) writing results to the standard output. The data files can be prepared in a format of either text or GeoTIFF, which is a widely used format in dealing with spatial raster data such as a landscape gradient. Note if the standard input or text file is chosen, one needs to prepare the landscape gradient in question as a matrix of numbers, as shown in Figure 5. The separator between two numbers can be a comma, space, 
or Tab. The second function is to compute all the fractal metrics of a landscape gradient. The last function is to compute the fractal metrics of a large number of landscape gradients in parallel.

There are three algorithmic considerations. First, the terms "small things" and "large ones" are interpreted as cells having a small value and the cells having a large value, respectively, when deriving a scaling pattern with landscape gradients. Second, the algorithms for computing fractal metrics should be capable of functioning based on different threshold percentages of small things. Third, the practical recommendations by Jiang and Ma [52] for computing the fht-index of a data set are followed in this study. The recommendations include (a) to determine the whole of a data set by appending small values that are computed based on the trend line of the data set and (b) to estimate the fht-index using a power interpolation between the ht-index of the whole of the data set and the ht-index of the largest sub-whole of the data set. Note that all these fractal metrics are theoretically computable even if a landscape gradient contains null values or is irregular in shape. This is because, as explained at the end of the second section, the new definition of fractal is focused on (non-null) values only.

The software tool was implemented with Microsoft Visual Studio 2015 Update 3 with Tools for Universal Windows Apps. The parallel computation of fractal metrics was carried out by using the Task Parallel Library in the "System.Threading.Tasks" namespace of NET Framework 4.5. The curve fitting (for the trend line of a data set) in computing fht-index was performed using the "Math.NET Numerics" (Available online: https://numerics.mathdotnet.com/), which is an open-source numerical library for applications developed in NET 4.0 and higher frameworks. The "read GeoTIFF" function was implemented using the Geospatial Data Abstraction Library (GDAL. Available online: https://gdal.org/). It can also be implemented using libraries such as LibTiff.Net (Available online: https://bitmiracle.com/libtiff) and Global Mapper SDK (Available online: https://www.bluemarblegeo.com/products/global-mapper-sdk.php). This software tool can be found in Supplementary Materials.

\section{Case Study and Analysis}

To illustrate how to use the developed tool, we present a case study of terrain analysis. Such analysis can be performed from diverse aspects, such as correlation [53] and disorder [54,55]. In this case study, we aimed to characterize the fractality of digital terrain data. Digital terrain data are widely used gradients in landscape ecology e.g., $[56,57]$ and are usually represented as digital elevation models (DEMs). In this case study, we obtained four pairs of DEMs from Gao, et al. [58], as shown in Figure 6. These DEMs were originally derived from NASA Shuttle Radar Topography Mission data (Available online: http://srtm.csi.cgiar.org/). The statistics of these DEMs were listed in Table 3.

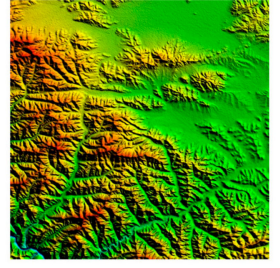

(A1)

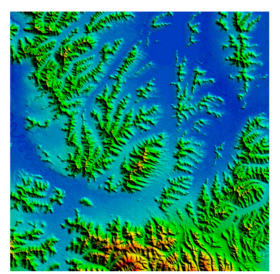

(C1)

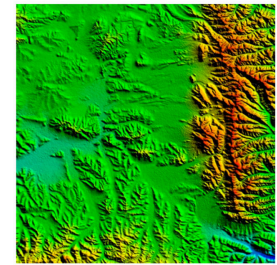

(A2)

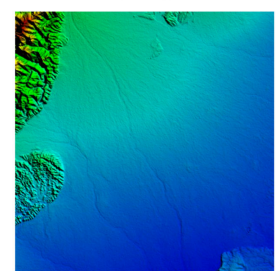

(C2)

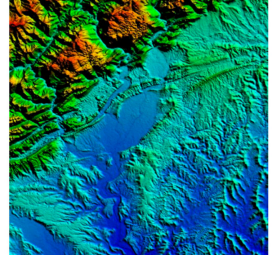

(B1)

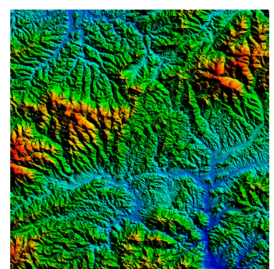

(D1)

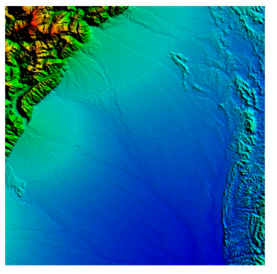

(B2)

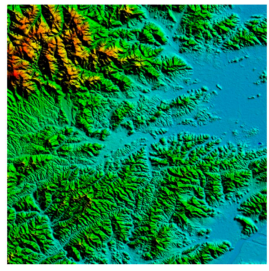

(D2)

Figure 6. Four pairs of digital elevation models (DEMs) [51], namely A1 and A2, B1 and B2, C1 and C2, and D1 and D2. 
Table 3. Statistics of the digital elevation models (DEMs) for experiments.

\begin{tabular}{cccccc}
\hline DEM & Size & Minimum & Maximum & Difference $^{\mathbf{1}}$ & Average $^{\text {M }}$ \\
\hline A1 & $600 \times 600$ & 3247 & 4473 & 1226 & 3765 \\
A2 & $600 \times 600$ & 3394 & 4380 & 986 & 3717 \\
\hline B1 & $600 \times 600$ & 449 & 2327 & 1878 & 724 \\
B2 & $600 \times 600$ & 453 & 2498 & 2045 & 634 \\
\hline C1 & $600 \times 600$ & 3428 & 4042 & 614 & 3511 \\
C2 & $600 \times 600$ & 460 & 2405 & 1945 & 593 \\
\hline D1 & $600 \times 600$ & 35 & 1589 & 1554 & 413 \\
D2 & $600 \times 600$ & -74 & 1460 & 1534 & 210 \\
\hline
\end{tabular}

Before using the FracL, we derived the elevations of each DEM as a text file. Then, we opened FracL and read a text file by clicking on the button "Read text file". Last, we computed all the fractal metrics by clicking on the button "Compute metrics". The computation results are shown in Table 4 . Note that all the text files can be found in Supplementary Materials.

Table 4. Fractal metrics of the digital elevation models (DEMs) for experiments.

\begin{tabular}{ccccccc}
\hline DEM & Ht-Index & CRG Index & RA Index & UM1 & UM2 & Fht-Index \\
\hline A1 & 13 & 11.1690 & 0.8449 & 13.0397 & 13.5477 & N/A \\
A2 & 14 & 12.1620 & 0.6553 & 14.0415 & 14.6221 & N/A \\
\hline B1 & 15 & 14.3706 & 0.2933 & 15.4017 & 15.7626 & N/A \\
B2 & 11 & 10.5855 & 0.1773 & 11.4036 & 11.8253 & N/A \\
\hline C1 & 12 & 10.1361 & 0.2713 & 12.0249 & 12.6566 & N/A \\
C2 & 13 & 12.5595 & 0.1376 & 13.1656 & 13.6623 & N/A \\
\hline D1 & 15 & 14.5958 & 0.4866 & 15.4144 & 15.6224 & N/A \\
D2 & 13 & 13.4606 & 0.3714 & 13.5256 & 13.6558 & N/A \\
\hline
\end{tabular}

The previous study [58] has demonstrated that in each pair, the first DEM is more disorderly (has a greater Boltzmann entropy) than the second one in terms of the disorder. However, in terms of fractality, not all the first DEMs are more fractal than the second one, as shown in Table 4. Specifically, according to $\mathrm{Ht} / \mathrm{CRG} / \mathrm{UM} 1 / \mathrm{UM} 2$, DEM A1 is less fractal than A2, B1 is more fractal than B2, C1 is less fractal than $\mathrm{C} 2$, and D1 is more fractal than D2. According to the RA index, the first DEM of each pair is more fractal than the second one. Fht-index is not computable with all DEMs. In summary, in this case, Ht-index, CRG, UM1, and UM2 are consistent with each other. None of them is consistent with the order/disorder of a DEM, indicating their difference from entropic measures.

Careful readers may note that the results shown by the RA index are not consistent with that by $\mathrm{Ht} / \mathrm{CRG} / \mathrm{UM} 1 / \mathrm{UM} 2$. This is because, as explained in Section 3.3, the RA index is not computed by performing the head/tail breaks method, while all the other metrics are. We argue that the RA index is not a suitable metric of the fractal under the new definition because it does not capture the similarity among different scales (i.e., hierarchical levels). It is only an indicator of the geometric shape of the pattern displayed by a dataset in a rank-size plot. Fht-index is not computable because the "whole" cannot be derived. This fact suggests that fht-index is theoretically appealing but experimentally difficult to compute.

\section{Discussion}

Several comparative and analytical studies immediately come to mind in thinking about the next steps in utilizing this new tool to explore fractality. First, it would be very interesting to compare the 
predictions of these new approaches with those produced by traditional fractal metrics in landscape ecology. The traditional fractal metrics widely used in landscape ecology are implemented on categorical patch mosaics, and the methods presented here are applied to gradient surfaces. However, modification of the definition of small and large objects could enable this tool to be applied to patch mosaics. Specifically, defining small and large objects in terms of the size distribution of patches in a landscape would enable the computation of these metrics and direct comparison to traditional landscape metrics like perimeter-area fractal dimension, PAFRAC [7]. This kind of direct comparison of the new fractal metrics with the existing ones across ranges of landscape pattern and complexity would help to understand both the behavior e.g., [59] and parsimony e.g., [60] of fractal measures of landscape structure.

Second, these new measures of fractal structure of surfaces provide a potentially important advance in the contemporary challenge of developing quantitative tools for analysis of the structure and complexity of surfaces. The gradient paradigm in landscape ecology depends on the maturation of such methods for its advancement. Few papers have been produced to date evaluating and comparing surface-based metrics for analyzing landscapes e.g., [10]. As such, a second important project would be to apply these gradient measures to a wide range of landscape gradients with different structures and compare them to the behavior and parsimony of the families of existing surface metrics e.g., [10]. In this context, it would also be informative to vary the criteria used to define small vs large objects. In the current conception presented in this paper, we use the value of a cell on a surface as a measure of "size". However, this has some apparent limitations in the context of the many small and few large objects theory behind this measure of fractality. Specifically, a highly concave landscape, such as one with peaks above generally lower values on the surface meets the expectation of many small and few large values. However, in the converse case, such as pits and valleys on a plateau surface, there will be many large values and relatively few small values. It will be important to see how these methods perform across this range of convexity vs. concavity of landscape gradient surfaces. This might also suggest reformulations using different definitions of large vs small objects on surfaces, such as the size of peaks in terms of their breadth rather than the value of cells per se.

It is of interest to note one recent approach to interpret the fractal metrics. The value of these fractal metrics has been interpreted as the degree of wholeness [61], which is conceptualized by the architect Christopher Alexander as a kind of structure of physical space [62]. It has been argued that the greater the fractal metric of a structure has, the more sustainable the structure is [63].

\section{Conclusions}

Fractal analysis is among the most widely used methods for characterizing landscape patterns and understand landscape dynamics. It is commonly performed based on the Mandelbrot [18] definition of fractal, which is "too strict for many geographic features" [19] and thus limits the potential of fractal analysis in landscape ecology. This study presents a software tool to facilitate the computation of metrics developed under a new definition of fractal. We hope this software tool will contribute to the understanding of landscape dynamics from a new perspective and help advance the development of the new gradient paradigm in landscape ecology.

Supplementary Materials: The following are available online at http://www.mdpi.com/2220-9964/8/10/466/s1.

Author Contributions: P.G. and G.L. developed the software tool; P.G. and S.A.C. wrote the paper; S.Y., S.S., and C.C. carried out the case study.

Funding: This research was funded by National Natural Science Foundation of China (Grant No. 41771537, 41901316, 41871303; 41801300), National Key Research and Development Plan of China (Grant No. 2017YFB0504102), and the Fundamental Research Funds for the Central Universities.

Acknowledgments: We would like to thank the four reviewers for their constructive comments, Jicheng Wang for his great help in using the GDAL library, Ding Ma for his helpful discussion on fractal metrics at the beginning of 2018, and the high-performance computing support from the Center for Geodata and Analysis, Faculty of Geographical Science, Beijing Normal University (Available online: https://gda.bnu.edu.cn/). 
Conflicts of Interest: The authors declare no conflict of interest.

\section{References}

1. Arnot, C.; Fisher, P.F.; Wadsworth, R.; Wellens, J. Landscape metrics with ecotones: Pattern under uncertainty. Landsc. Ecol. 2004, 19, 181-195. [CrossRef]

2. Frazier, A.E.; Wang, L. Modeling landscape structure response across a gradient of land cover intensity. Landsc. Ecol. 2013, 28, 233-246. [CrossRef]

3. Forman, R.T.T. Land Mosaics: The Ecology of Landscapes and Regions; Cambridge University Press: Cambridge, UK, 1995.

4. McGarigal, K.; Cushman, S.A. The gradient concept of landscape structure. In Issues and Perspectives in Landscape Ecology; Wiens, J.A., Moss, M.R., Eds.; Cambridge University Press: Cambridge, UK, 2005; pp. 112-119. [CrossRef]

5. Cushman, S.A.; Evans, J.S.; McGarigal, K. Landscape ecology: Past, present, and future. In Spatial Complexity, Informatics, and Wildlife Conservation; Cushman, S.A., Huettmann, F., Eds.; Springer: Tokyo, Japan, 2010; pp. 65-82.

6. Evans, J.S.; Cushman, S.A. Gradient modeling of conifer species using random forests. Landsc. Ecol. 2009, $24,673-683$. [CrossRef]

7. McGarigal, K.; Cushman, S.A.; Eduard, E. FRAGSTATS v4: Spatial Pattern Analysis Program for Categorical and Continuous Maps. Available online: http:/www.umass.edu/landeco/research/fragstats/fragstats.html (accessed on 17 January 2019).

8. Costanza, J.K.; Riitters, K.; Vogt, P.; Wickham, J. Describing and analyzing landscape patterns: Where are we now, and where are we going? Landsc. Ecol. 2019, 34, 2049-2055. [CrossRef]

9. Gustafson, E.J. How has the state-of-the-art for quantification of landscape pattern advanced in the twenty-first century? Landsc. Ecol. 2019, 34, 2065-2072. [CrossRef]

10. McGarigal, K.; Tagil, S.; Cushman, S.A. Surface metrics: An alternative to patch metrics for the quantification of landscape structure. Landsc. Ecol. 2009, 24, 433-450. [CrossRef]

11. Kedron, P.J.; Frazier, A.E.; Ovando-Montejo, G.A.; Wang, J. Surface metrics for landscape ecology: A comparison of landscape models across ecoregions and scales. Landsc. Ecol. 2018, 33, 1489-1504. [CrossRef]

12. Frazier, A.E. Surface metrics: Scaling relationships and downscaling behavior. Landsc. Ecol. 2016, 31, 351-363. [CrossRef]

13. Smith, A.C. Bioxgeo/geodiv: Methods for Calculating Gradient Surface Metrics. Available online: https: //rdrr.io/github/bioXgeo/geodiv/ (accessed on 9 September 2019).

14. Koch, H. Sur une courbe continue sans tangente, obtenue par une construction géométrique élémentaire [On a continuous curve without tangents constructible from elementary geometry]. Arkiv för Matematik 1904, 1, 681-704.

15. Sierpinski, W. Sur une courbe cantorienne qui contient une image biunivoque et continue de toute courbe donnée [On a cantorian curve which contains a continuous one-to-one image of every given curve]. Comptes Rendus 1916, 162, 629-632.

16. Menger, K. Allgemeine Räume und Cartesische Räume., I. Proc. Amst. 1927, 29, 476-482.

17. Mandelbrot, B.B. Fractals: Form, Chance, and Dimension; Freeman: San Francisco, CA, USA, 1977.

18. Mandelbrot, B.B. How long is the coast of Britain? Statistical self-similarity and fractional dimension. Science 1967, 156, 636-638. [CrossRef] [PubMed]

19. Jiang, B.; Yin, J.J. Ht-index for quantifying the fractal or scaling structure of geographic features. Ann. Assoc. Am. Geogr. 2014, 104, 530-540. [CrossRef]

20. Vranken, I.; Baudry, J.; Aubinet, M.; Visser, M.; Bogaert, J. A review on the use of entropy in landscape ecology: Heterogeneity, unpredictability, scale dependence and their links with thermodynamics. Landsc. Ecol. 2015, 30, 51-65. [CrossRef]

21. Parrott, L.; Proulx, R.; Thibert-Plante, X. Three-dimensional metrics for the analysis of spatiotemporal data in ecology. Ecol. Inform. 2008, 3, 343-353. [CrossRef]

22. Jiang, B. Head/tail breaks: A new classification scheme for data with a heavy-tailed distribution. Prof. Geogr. 2013, 65, 482-494. [CrossRef]

23. Ma, D.; Jiang, B. A smooth curve as a fractal under the third definition. Cartogr. Int. J. Geogr. Inform. Geovis. 2018, 53, 203-210. [CrossRef] 
24. Mac Carron, P.; Kaski, K.; Dunbar, R. Calling Dunbar's numbers. Soc. Netw. 2016, 47, 151-155. [CrossRef]

25. Long, Y.; Shen, Y.; Jin, X.B. Mapping block-level urban areas for all Chinese cities. Ann. Am. Assoc. Geogr. 2016, 106, 96-113. [CrossRef]

26. Krummel, J.; Gardner, R.; Sugihara, G.; O'neill, R.; Coleman, P. Landscape patterns in a disturbed environment. Oikos 1987, 48, 321-324. [CrossRef]

27. Milne, B.T. Measuring the fractal geometry of landscapes. Appl. Math. Comput. 1988, 27, 67-79. [CrossRef]

28. Nams, V.O. The VFractal: A new estimator for fractal dimension of animal movement paths. Landsc. Ecol. 1996, 11, 289-297. [CrossRef]

29. Zhang, Y.X.; Ma, K.M.; Anand, M.; Fu, B.J. Multifractal pattern and process during a recent period of forest expansion in a temperate mountainous region of China. Ecol. Inform. 2011, 6, 384-390. [CrossRef]

30. Gao, P.C.; Liu, Z.; Liu, G.; Zhao, H.R.; Xie, X.X. Unified metrics for characterizing the fractal nature of geographic features. Ann. Am. Assoc. Geogr. 2017, 107, 1315-1331. [CrossRef]

31. Jiang, B. Computing the image of the city. In Planning Support Tools: Policy Analysis, Implementation and Evaluation (Proceedings of the 7th International Conference on Informatics and Urban and Regional Planning INPUT2012); Campagna, M., Montis, A.D., Isola, F., Lai, S., Pira, C., Zoppi, C., Eds.; FrancoAngeli: Milan, Italy, 2012; pp. 111-121.

32. Robinson, A.C.; Demšar, U.; Moore, A.B.; Buckley, A.; Jiang, B.; Field, K.; Kraak, M.-J.; Camboim, S.P.; Sluter, C.R. Geospatial big data and cartography: Research challenges and opportunities for making maps that matter. Int. J. Cartogr. 2017, 3, 32-60. [CrossRef]

33. Mainuri, Z.G.; Owino, J.O. Spatial variability of soil aggregate stability in a disturbed river watershed. Eur. J. Econ. Bus. Stud. 2017, 9, 278-290. [CrossRef]

34. Chen, Y.G. Urban chaos and replacement dynamics in nature and society. Phys. A Stat.Mech. Appl. 2014, 413, $373-384$. [CrossRef]

35. He, J.; Zhang, H.; Lan, T.; Cao, W.W.; Wu, X. Exploring the hierarchical structure in road network. In Proceedings of the International Conference on Intelligent Earth Observing and Applications, Guilin, Guangxi, China, 9 December 2015.

36. Ma, D.; Sandberg, M.; Jiang, B. A socio-geographic perspective on human activities in social media. Geogr. Anal. 2017, 49, 328-342. [CrossRef]

37. McNutt, S.R.; Roman, D.C. Volcanic seismicity. In The Encyclopedia of Volcanoes, 2nd ed.; Elsevier: Amsterdam, The Netherlands, 2015; pp. 1011-1034.

38. Sage, L.J. Second light: The biggest stars. J. R. Astron. Soc. Can. 2005, 99, 59.

39. Wu, J.G. Effects of changing scale on landscape pattern analysis: Scaling relations. Landsc. Ecol. 2004, 19, 125-138. [CrossRef]

40. Liu, G.; Li, R.; He, J.; Li, W.L.; Lu, J.Y.; Long, W.; Gao, P.C.; Cai, G.L.; Tang, M. Scaling relation of earthquake seismic data. Phys. A Stat. Mech. Appl. 2018, 492, 2092-2102. [CrossRef]

41. Zhang, H.; Li, Z.L. Fractality and self-similarity in the structure of road networks. Ann. Assoc. Am. Geogr. 2012, 102, 350-365. [CrossRef]

42. Lan, T.; Li, Z.L.; Zhang, H. Urban allometric scaling beneath structural fractality of road networks. Ann. Assoc. Am. Geogr. 2019, 109, 943-957. [CrossRef]

43. Huang, K.Y. Fractal or Scaling Analysis of Natural Cities Extracted from Open Geographic Data Sources. Ph.D. Thesis, University of Gävle, Gävle, Sweden, 2015.

44. Wu, W.J.; Zhao, H.R.; Jiang, S.L. A Zipf's law-based method for mapping urban areas using NPP-VIIRS nighttime light data. Remote Sens. 2018, 10, 1-24. [CrossRef]

45. Long, Y.; Zhai, W.X.; Shen, Y.; Ye, X.Y. Understanding uneven urban expansion with natural cities using open data. Landsc. Urban Plann. 2017, 177, 281-293. [CrossRef]

46. Liu, Z.; Xie, M.H.; Tian, K.; Xie, X.X. Classification of PM2. 5 for natural cities based on co-Kriging and head/tail break algorithms. J. Tsinghua Univ. Sci. Technol. 2017, 57, 555-560.

47. Li, X.; Lv, Z.H.; Zheng, Z.G.; Zhong, C.; Hijazi, I.H.; Cheng, S.D. Assessment of lively street network based on geographic information system and space syntax. Multimed. Tools Appl. 2017, 76, 17801-17819. [CrossRef]

48. O'Neill, R.V.; Johnson, A.R.; King, A.W. A hierarchical framework for the analysis of scale. Landsc. Ecol. 1989, 3, 193-205. [CrossRef]

49. O'Neill, R.V.; Deangelis, D.L.; Waide, J.B.; Allen, T.F.; Allen, G.E. A Hierarchical Concept of Ecosystems; Princeton University Press: Princeton, NJ, USA, 1986. 
50. Gao, P.C.; Liu, Z.; Xie, M.H.; Tian, K.; Liu, G. CRG index: A more sensitive ht-index for enabling dynamic views of geographic features. Prof. Geogr. 2016, 68, 533-545. [CrossRef]

51. Gao, P.C.; Liu, Z.; Tian, K.; Liu, G. Characterizing traffic conditions from the perspective of spatial-temporal heterogeneity. ISPRS Int. J. Geo-Inform. 2016, 5, 34. [CrossRef]

52. Jiang, B.; Ma, D. How complex is a fractal? Head/tail breaks and fractional hierarchy. J. Geovis. Spatial Anal. 2018, 2, 6. [CrossRef]

53. Da Silva, J.M.F.; Santos, L.J.C.; Oka-Fiori, C. Spatial correlation analysis between topographic parameters for defining the geomorphometric diversity index: Application in the environmental protection area of the Serra da Esperança (state of Paraná, Brazil). Environ. Earth Sci. 2019, 78, 356. [CrossRef]

54. Gao, P.C.; Li, Z.L. Computation of the Boltzmann entropy of a landscape: A review and a generalization. Landsc. Ecol. 2019, 34, 2183-2196. [CrossRef]

55. Cushman, S.A. Calculation of configurational entropy in complex landscapes. Entropy 2018, 20, 298. [CrossRef]

56. Ollinger, S.V.; Aber, J.D.; Federer, A. Estimating regional forest productivity and water yield using an ecosystem model linked to a GIS. Landsc. Ecol. 1998, 13, 323-334. [CrossRef]

57. Bond, M.L.; Bradley, C.M.; Kiffner, C.; Morrison, T.A.; Lee, D.E. A multi-method approach to delineate and validate migratory corridors. Landsc. Ecol. 2017, 32, 1705-1721. [CrossRef]

58. Gao, P.C.; Zhang, H.; Li, Z.L. A hierarchy-based solution to calculate the configurational entropy of landscape gradients. Landsc. Ecol. 2017, 32, 1133-1146. [CrossRef]

59. Neel, M.C.; McGarigal, K.; Cushman, S.A. Behavior of class-level landscape metrics across gradients of class aggregation and area. Landsc. Ecol. 2004, 19, 435-455. [CrossRef]

60. Cushman, S.A.; McGarigal, K.; Neel, M.C. Parsimony in landscape metrics: Strength, universality, and consistency. Ecol. Indic. 2008, 8, 691-703. [CrossRef]

61. Jiang, B.; Ren, Z. Geographic space as a living structure for predicting human activities using big data. Int. J. Geogr. Inf. Sci. 2019, 33, 764-779. [CrossRef]

62. Alexander, C. The Nature of Order: An Essay on the Art of Building and The Nature of the Universe; The Center for Environmental Structure: Berkeley, CA, USA, 2002.

63. Jiang, B. A recursive definition of goodness of space for bridging the concepts of space and place for sustainability. Sustainability 2019, 11, 4091. [CrossRef] 